\title{
Costimulation to enhance the antitumor activity of CD19 eng T cells
}

\author{
Mireya Velasquez ${ }^{1 *}$, David Torres ${ }^{2}$, Aarohi Thakkar ${ }^{1}$, Sunitha Kakarla ${ }^{1}$ Carolyn Arber Barth ${ }^{1}$, Xiao-Tong Song ${ }^{2}$, \\ Stephen Gottschalk'
}

From 30th Annual Meeting and Associated Programs of the Society for Immunotherapy of Cancer (SITC 2015) National Harbor, MD, USA. 4-8 November 2015

\section{Background}

Immunotherapy with $\mathrm{T}$ cells or bispecific $\mathrm{T}$ cell engagers is one promising approach to improve outcomes for patients with CD19-positive hematological malignancies. We had previously shown that $\mathrm{T}$ cells expressing bispecific $\mathrm{T}$ cell engagers that recognize CD19 and CD3 (CD19-ENG $T$ cells) are activated in an antigen dependent manner, recruit resident $\mathrm{T}$ cells to tumors, and have anti-tumor activity in preclinical models. In this project we now wanted to evaluate if provision of co-stimulation enhances the effector function of CD19-ENG T cells by expressing CD80 and 41BBL on their cell surface (CD19 ENG/costim $\mathrm{T}$ cells).

\section{Methods}

CD19-ENG $T$ cells were generated by transducing $T$ cells with a retroviral vector encoding a CD19-specific T cell engager, and CD19-ENG/Costim T cells were generated by double transducing $\mathrm{T}$ cells with the previous construct and a $2^{\text {nd }}$ retroviral vector encoding the costimulatory molecules 41BBL and CD80. The effector function of the generated $\mathrm{T}$ cells was evaluated in vitro and in a xenograft model.

\section{Results}

CD19-ENG and CD19-ENG/Costim T cells recognized CD19+ lymphoma (Daudi, Raji) and acute leukemia (BV173) cells as judged by IFN-g secretion. Both ENG $\mathrm{T}$ cell populations produced IL-2 in the presence of CD19-positive targets expressing CD80 and CD86 (Daudi and Raji). However, CD19-ENG/Costim T cells produced higher levels of IL-2 in comparison to CD19-ENG T cells after stimulation with BV173
(CD19+CD80-CD86-). ENG and ENG/Costim T cells specific for an irrelevant antigen (EphA2) did not produce cytokines, confirming antigen dependence. Specificity was confirmed in cytotoxicity assays. In vivo antitumor activity of CD19-ENGand CD19-ENG/Costim $\mathrm{T}$ cells was assessed in a BV173/NSG xenograft model. First we determined the minimal required CD19-ENG $\mathrm{T}$ cell dose to observe anti-tumor effects. Three injections of $1 \times 10^{7}$ CD19-ENG $\mathrm{T}$ cells cured $5 / 5$ mice, one injection 2/5 mice, and one dose of $1 \times 10^{6}$ CD19-ENG $\mathrm{T}$ cells had no anti-tumor effects. In contrast one dose of $1 \times 10^{6}$ or $1 \times 10^{7}$ CD19-ENG/Costim T cells cured 5/5 mice. ENG $\mathrm{T}$ cells or ENG/Costim $\mathrm{T}$ cells recognizing EphA2 had no anti-tumor effects.

\section{Conclusions}

Provision of co-stimulation increases the effector function of CD19-ENG $T$ cells resulting in enhanced anti-tumor activity in vivo. Genetically modifying $\mathrm{T}$ cells to express engagers and additional molecules to enhance their effector function may present a promising alternative to current CD19-targeted immunotherapies.

\section{Authors' details \\ 'Baylor College of Medicine, Center for Cell and Gene Therapy, Houston, TX,} USA. 'Baylor College of Medicine, Houston, TX, USA.

Published: 4 November 2015

doi:10.1186/2051-1426-3-S2-P58

Cite this article as: Velasquez et al.: Costimulation to enhance the antitumor activity of CD19 eng T cells. Journal for ImmunoTherapy of Cancer 2015 3(Suppl 2):P58. 